SCHIAWE, L.: Zur geographischen Verbreitung der Ginsterkatzen, Gattung Genetta G. Cuvier, 1816 (Mammalia, Carnivora, Viverridae). 3 Fundortkarten (thematische Computer-Kartographie, Programmsystem "Thekar").

On the Geographic Distribution of Genets, Genus Genetta G. Cuvier, 1816.

Nr. Seiten

$147-16]$

VESMANIS, I. E. u. A. VESMANIS: Ein Nachweis des Siebenschläfers, Glis glis (Linnaeus, 1766) aus Eulengewöllen von der Insel Elba, Italien (Mammalia, Rodentia, Muscardinidae). 1 Abb.

A Record of the Edible Doormouse, Glis glis (Linnaeus, 1766), from Owl Pellets of the Mediterranean Island of Elba (Italy).

$167-170$

\title{
FAUNISTISCHE KURZMITTEILUNGEN
}

ARNOLD, A.: Zum Vorkommen von Lurchen in Höhlen, Stollen und Bergwerken im Westerzgebirge (Amphibia).

$145-146$

BISCHOFF, W. u. W.-E. ENGELMANN: Zur aktuellen Entwicklung der Smaragdeidechsen-Population von Lieberose $\mathrm{Kr}$. Beeskow (Reptilia, Lacertidae).

DOLCH, D.: Zwei neue Vorkommen der Kleinäugigen Wühlmaus, Pitymps subterraneus (De Selys-Longchamps, 1836) im Norden der DDR (Mammalia, Rodentia).

$139-140$

EMMRICH, R.: Vorkommen von Cacopsplla hippophaes (Frst.) und C. zetterstedti (Thoms.) auf Hiddensee (Bez. Rostock) (Homoptera, Psyllodea).

KRAUSS, A.: Notizen über ein Hausrattenvorkommen in Einsiedel bei Karl-Marx-Stadt (Manmalia, Rodentia).

$121-122$

KRAUSS, A.: Ein weiterer Nachweis von Eptesicus serotinus (Schreber) für den Bezirk Karl-Marx-Stadt (Mammalia, Chiroptera).

WILHELM, M. u. Z. BARTA: Eine Luchsfährte im böhmischen Teil des Elbsandsteingebirges (Mammalia, Felidae).

$23-24$

Neue Namen, Synonyme und Kombinationen

ARACHNIDA

Theridiidae

Chrysso conigerum (SIMON, 1914) comb. n., p. 179: HEIMER

\section{COLEOPTERA}

Alleculidae

Omophlina muminovi sp. n., p. 291: MUCHE

Anthicidae

Anthicus dzhungaricus sp. n., p. 142: MEDVEDEV et MICHEETCHEV

Chrysomelidae

Afrophthalma gen. n., p. 57: MEDVEDEV

Afrophthalma elongata (JACOBY) comb. n., p. 57 - A. filiformis (LACORDAIRE) comb. n., p. 57 - A. apicalis (JACOBY) comb. n., p. 57 - A. lefevrei (CHAPUIS) comb. n., p. 57 - A. lefeurei nigrosuturalis ssp. n., p. 57 - A. zanzibarica (LEFEVRE) comb. n., p. 58: MEDVEDEV

Gqnandrophthalma (Smaragdina) weisei JACOBY, sYn. n. zu Smaragdina immaculata LACORDAIRE, p. 56: MEDVEDEV 
Melitonoma ghanensis sp. n., p. 58: MEDVEDEV

Paraclytra crocata (LACORDAIRE) comb. n., p. 55: MEDVEDEV

Platypodidae

Diapus apertus sp. n., p. 119 - D. latespinis sp. n., p. 120: SCHEDL

Platypus ponomae SCHEDL, sYn. n. zu Pl. apicatus SCHEDL, p. 96 - Pl. ustus SCHEDL, syn. n. zu Pl. pilidens SCHEDL, p. $96-$ Pl. artetruncatus sp. n., p. $109-\mathrm{Pl}$. collaris sp. n., p. 110 - Fl. depressus sp. n., p. 110 - Pl. duplicatus sp. n., p. 111 Pl. extensus sp. n., p. $111-$ Pl. filipennis sp. n., p. $111-$ Pl. frontebulbifer sp. n., p. 112 - Pl. globicollis sp. n., p. 112 - Pl. lablabiae sp. n., p. 113 - Pl. lucaris sp. n., p. 114 - Pl. micrographus sp. n., p. 114 - Pl. neotruncatus sp. n., p. 114 - Pl. paradoxus sp. n., p. 115 - Pl. plumatus sp. n., p. 116 - Pl. praetermissus sp. n., p. 117 - Pl. pseudoporus sp. n., p. 117 - Pl. rasilis sp. n., p. 118 - Pl. sulcaticeps sp. n., p. 118 - Pl. transversus sp. n., p. 119 - Pl. scalaris sp. n., p. 188: SCHEDL

Scarabaeidae

Lasiopsis (Brahmina) medoedevi sp. n., p. 224 - L. (Brahmina) glabellus sp. n., p. 225 L. (Brahmina) kabulicus sp. n., p. $226-$ L. (Brahmina) kandaharicus sp. n., p. 227 L. (Brahmina) nurestanicus sp. n., p. 227: NIKOLAJEV et KABAKOV

Lasiopsis (s. str.) krpzhanouskii sp. n., p. 223: NIKOLAJEV et KABAKOV

Miltotrogus nonveilleri sp. n., p. 227: NIKOLAJEV et KABAKOV

Scolytidae

Arixpleborus sus (SCHEDL) comb. n., p. 95 - A. varicus SCHEDL, syn. n. zu A. sus (SCHEDL), p. $95-A$. deceptus sp. n., p. 103: SCHEDL

Coccotrppes omissus sp. n., p. 101: SCHEDL

Crpphalomorphus ankius sp. n., p. $96-$ Cr. excellens sp. n., p. $97-$ Cr. papuanus sp. n., p. 97: SCHEDL

Cryphalus planicollis sp. n., p. $74-\mathrm{Cr}$. tetricus (SCHEDL) comb. n., p. $95-\mathrm{Cr}$. erraticum sp. n., p. $96-$ Cr. papuanus SCHEDL, syn. n. zu Cr. tetricus (SCHEDL), p. $95-$ Cr. grapi SCHEDL, syn. n. zu Cr. tetricus (SCHEDL), p. 95: SCHEDL

Eidophelus elongatus sp. 11., p. $102-$ E. subsulcatus sp. n., p. $102-$ E. sulcatus sp. n., p. 103: SCHEDL

Hypocrpphalus pilifer sp. n., p. 98: SCHEDL

Lepiceroides spinosus sp. n., p. 98: SCHEDL

Ozodendron aequalis sp. n., p. 100: SCHEDL

Peridrpocoetes squamipennis sp. n., p. 101: SCHEDL

Poecilips abruptus sp. 11., p. $99-$ P. fulgens sp. n., p. 99: SCHEDL

X'pleborus atavus sp. n., p. $104-X$. bispinus sp. n., p. $104-X$ cinctipes sp. n., p. $105-$ $X$. densatus sp. n., p. $106-X$. devius sp. n., p. $106-X$. flavipennis sp. n., p. $107-$ $X$. funestus sp. n., p. $107-X$. mesoleinlus sp. n., p. $108-X$. mixtus sp. n., p. $108-$ $X$. australis sp. n., p. $185-X$. cinctipennis sp. n., p. 186: SCHEDL

\section{Staphylinidae}

Paederus kamonoensis sp. n., p. $123-$ P. wauensis sp. n., p. $124-P$. okapaensis sp. n., p. $125-$ P. reticulatus sp. n., p. $125-$ P. copiosus sp. n., p. $126-P$. buluensis sp. n., p. $126-$ P. kairdiensis sp. n., p. $127-$ P. michaelensis sp. n., p. $127-P$. katubuensis sp. n., p. $128-P$. huonensis sp. n., p. $128-$ P. cameroni sp. n., $129-$ $P$. perlongus sp. n., p. $131-P$. hornabrooki sp. n., p. $133-P$. mimicus sp. n., p. 133 - P. chimbuensis sp. n., p. $134-$ P. aequabilis sp. n., p. $134-$ P. hagenensis sp. n., p. 135 - P. problematis sp. n., p. 136 - P. laensis sp. n., p. 136 P. mafuluensis sp. n., p. $137-$ P. fuscus sp. n., p. 137: LAST

Megachilidae

HYMENOPTERA

Hoplitis (s. str.) heinrichi sp. n., p. 234: VAN DER ZANDEN

Tenthredinidae

Macrophra punctumalbum maroccana ssp. n., p. 92: MUCHE

Phryganeidae

\section{TRICHOPTERA}

Semblis atrata chinganica MARTYNOV stat. n., p. 173: MEY et DULMAA 
Holcopogonidae

\section{LEPIDOPTERA}

Hesperesta gen. n., p. 61: GOZNIANY

Hesperesta geminella (CHRÉTIEN, 1915) comb. n., p. 62: GOZMANY

Plutellidae

Ypsolopha bigraphella (TURATI, 1929) comb. n., p. 62: GOZMANY

\section{Berichtigungen}

Nr. 1 (MARTIN), S. 1: Der Titel der Arbeit mu\& korrekt lauten: „Zur Verbreitung der Zebraspinne (Argiope bruennichi [SCOP.]) in der DDR".

Nr. 6 (MEDVEDEV), S. 58: Die Zeilen 30 u. 31 dieser Seite (in der Beschreibung von Melitonoma ghanensis sp. $\mathrm{n}$.) müssen wie folgt lauten:

$"$ "...holotype (male) and 5 paratypes; Northern region, Tamale (N $\left.8^{0} 25^{\prime}-\mathrm{W} 0^{0} 53^{\circ}\right), 24$. IX. 1971, netted, No. 478, 1 paratype; Northern region, Yendy $\left(\mathrm{N}^{0} 25^{\prime}-\mathrm{E} 0^{\circ} 00^{\circ}\right), \ldots$.

Nr. 8 (RÚSSEL), S. 68: Die letzten 11 Zeilen dieser Seite (von unten) wurden vor dem Druck verstellt. Sie müssen in richtiger Reihung wie folgt lauten:

„Das entspricht einem Zahlenverhältnis von durchschnittlich 3,75 o $\sigma^{*} \mathrm{zu} 1$ \%. Ein ähnliches großes Ubergewicht männlicher Bartfledermäuse stellte HANAK (1971) auch für das Winterquartier der Dobschauer Eishöhle in der Slowakei fest. Die Frage ist nun: Uberwintern die weiblichen Tiere an anderen Orten oder entspricht das festgestellte Zahlenverhältnis der Geschlechter im Winterquartier der realen Zusammensetzung der Populationen?

Von den 450 beringten Bartfledermäusen wurden 90 Tiere bisher im Kalkwerk wiedergefunden. Das entspricht einer Wiederfundrate von $20 \%$. Die Wiederfunde bestärkten uns in der Vermutung, daß die Fledermäuse noch Ausweichquartiere haben müssen, denn in jedem Winter tauchen Tiere auf, die schon vor einigen Wintern hier beringt wurden, ohne daß sie bisher wiedergefunden worden waren. Aber auch der Umstand, daß bei..."

$(\mathrm{Ab} \mathrm{S} .147$ eventuell verbliebene, technologisch bedingte Fehler konnten hier nicht mehr zur Berichtigung aufgenommen werden.)

Verantwortlicher Redakteur: Dr. RAINER EMMRICH

Staatliches Museum für Tierkunde Dresden

DDR - 8010 Dresden, Augustusstraße 2

Genehmigt zum Druck: Ministerrat der Deutschen Demokratischen Republik, Ministerium für Kultur, unter Nr, 105/16/78 u. 105/11/79

Klischees: Graphischer Großbetrieb "Völkerfreundschaft" Dresden

Satz u. Druck: Graphische Werkstätten Zittau-Görlitz, BT V Druckerei Neugersdorf 1II-19-2 